\title{
Assessment of the Association between Neuropeptide Y and Chronic Kidney Disease Progression
}

\author{
Haitham Ezzat ${ }^{1}$, Saeed Abdel Wahab Saeed ${ }^{1}$, Hend Ahmed Abouelsaad ${ }^{2}$, Nahla Mohamed Teama*1 \\ ${ }^{1}$ Department of Internal Medicine and Nephrology, Faculty of Medicine-Ain Shams University \\ ${ }^{2}$ Internal Medicine - Ain Shams University \\ *Corresponding author: Dr. Nahla Mohamed Teama, Tel: (+20) 01090447009; ORCID: 0000-0003-0095-8721. \\ E-mail: nahlateama@med.asu.edu.eg
}

\begin{abstract} progression predictor for CKD.

\section{INTRODUCTION}

Neuropeptide Y (NPY) is a sympathetic neurotransmitter with wide-extending impacts in different systems, from the central nervous system (CNS) to the cardiovascular system (CVS), the bone and the kidney. Increased sympathetic activity was suspected to play a role in renal disease progression ${ }^{(1)}$. A sympathetic activity marker (e.g. pulse) predicts movement to kidney failure autonomously of other factors in older chronic kidney disease (CKD) patients. Coursing NPY levels have just been related with left ventricular hypertrophy ${ }^{(2)}$ and incident CV occasions ${ }^{(3)}$ and bone illness ${ }^{(4)}$ in advanced CKD.

Neuropeptide Y (NPY) is a vasoactive neuropeptide widely distributed in the central and peripheral nervous system. The gut and associated organs are likely to be the source of most circulating NPY, and the release of this substance is stimulated after feeding in a way that does not correlate with norepinephrine (NE) ${ }^{(5)}$. This neuropeptide is also co-released with NE during sympathetic nerve stimulation, and it is extensively involved in cardiovascular $(\mathrm{CV})$ regulation because it modulates heart rate, cardiac excitability, and ventricular function as well as coronary blood flow.

Sympathetic neural responses regulating the systemic circulation can be measured by the level of circulating NPY ${ }^{(5)}$. The increased sympathetic activity has its drawbacks on the renal function. It can, not only, increase a pre-existing renal impairment, but also cause
\end{abstract}

Background: Neuropeptide Y (NPY) is a sympathetic neurotransmitter with wide-ranging effects in various organ systems, from the central nervous system (CNS) to the cardiovascular system, the bone and the renal system. There is a strong association between serum concentration of NPY and deterioration of eGFR and proteinuria as suggested by recent studies, however, its real effect on chronic kidney disease (CKD) progression is uncertain.

Objective: This study was conducted to assess the relationship between serum NPY and CKD progression.

Materials and Methods: In this observational case-control study all participants were subjected to renal function tests and calculation of estimated glomerular filtration rate, urinary protein/creatinine ratio, serum NPY and pelviabdominal ultrasonography at baseline and repeated for the patients only after six months as follow up.

Results: Serum NPY increased with a statistically significant difference in the patients group after six months follow up, the same as serum creatinine which increased significantly after six months and eGFR decreased with statistically significant difference. Also there was high statistically significant rise in the blood urea. There was no statistically significant difference rise in protein creatinine ratio after six months. The ROC curve determined the sensitivity and specificity of serum NPY as a marker of progression in CKD patients and the best cut off point to detect CKD status was calculated with NPY greater than $110 \mathrm{ng} / \mathrm{L}$ with sensitivity $100 \%$ and specificity $93.33 \%$.

Conclusion: Serum NPY could be a useful sensitive and specific marker that can be used as diagnostic and

Keywords: Neuropeptide Y, Chronic kidney disease, Sympathetic neurotransmitter.

damage to a normal functioning kidney ${ }^{(6)}$. Moreover, it is previously reported that albuminuria and podocyte injury in the settings of high sympathetic activity and renin-angiotensin system (RAS) activation could be prevented by sympathetic denervation. GFR and with proteinuria in CKD patients are closely related with the measured sympathetic activity ${ }^{(7)}$. Some CKD patients based studies have also showed that sympathetic denervation was associated with hypertension control and GFR stabilization. Thus, there is emerging experimental evidence that high sympathetic activity plays a role in CKD progression ${ }^{(\mathbf{8})}$.

It is like a vicious circle, NPY has a vasoconstrictive effect on the renal vasculature causing decrease in renal blood flow and increase in renal vascular resistance ${ }^{(3)}$, and in the same time there is increased serum NPY due to increased NPY gene expression, decreased NPY excretion and many other factors all associated with or caused by CKD. This study was conducted to assess the relationship between serum NPY and CKD progression.

\section{MATERIALS AND METHODS}

This was an observational case-control study conducted in Ain Shams University Hospital in Cairo, Egypt. The study included 60 subjects; 30 CKD subjects (group I) stage 1 to 4 and 30 healthy subjects as a control group (group II). Group I was called ( $a$ and b) 
according to time of laboratory investigations (at baseline and follow up after six months, respectively).

All participants were subjected to: full medical history including (personal history including, age, sex and drug history), thorough clinical examination including (anthropometric measurements: weight, height and BMI calculation "BMI $=$ weight $(\mathrm{kg}) /$ height in meters ${ }^{2}$ ) and laboratory tests and imaging (all participants in the study were subjected to: complete blood count (WBC, $\mathrm{Hb}$, platelets), serum $\mathrm{Na}, \mathrm{K}$ $(\mathrm{mEq} / \mathrm{L})$, blood urea and serum creatinine $(\mathrm{mg} / \mathrm{dL})$, serum $\mathrm{Ca}$ and $\mathrm{PO}_{4}(\mathrm{mg} / \mathrm{dL})$, serum albumin $(\mathrm{g} / \mathrm{dL})$, eGFR $\left(\mathrm{ml} / \mathrm{min} / 1.73 \mathrm{~m}^{2}\right)$ calculated by MDRD equation, urine analysis and protein/creatinine ratio, serum NPY (ng/L) and pelvi-abdominal ultrasonography). All these investigations were done as follow up for the patients only after six months from the baseline.

All patients with acute or rapidly evolving renal disease, congestive heart failure at baseline, kidney transplantation, pregnancy, cancer or diseases in terminal phase, BMI $>30$, age less than 18 years old and age more than 75 years old were excluded from the study. The data were collected at the Nephrology Departments. Neuropeptide Y was measured with ELISA.

Ethics approval and consent to participate:

This study was performed obtained in accordance with the ethical standards of Ain Shams University Research Committee (committee's reference number
12/2019) and with the 1964 Helsinki Declaration and its later amendments or comparable ethical standards. A written informed consent was taken from all individual participants included in the study.

\section{Statistical analysis}

The statistical analysis of the data was carried out using the Excel system for data and the SPSS program version 17. In order to check the normality of data distribution, the K-S (Kolmogorov-Smirnov) test was done. Data are presented as means $( \pm)$ SD and range for quantitative data and in number and percentage for qualitative data. For the quantitative data, the independent Student t-test was used to compare the two classes and paired t-test was used to compare the same group at baseline and follow up after six months. Chi square test was used for qualitative data. Spearman correlation coefficient was used to check the relationship between variable. $\mathrm{P}$ was considered significant if $<$ or $=0.05$ at 95 percent confidence interval.

\section{RESULTS}

Sixty subjects were included in the study; 28 (46.67\%) male and $32(53.33 \%)$ female. 23 patients were hypertensive and 16 were diabetic.

The primary renal diseases in patients' group were as follow: $53.33 \%$ diabetes, $6.67 \%$ hypertension, $10 \%$ glomerulonephritis, $6.67 \%$ congenital and $6.67 \%$ autoimmune.

Table (1): Comparison between group I and group II as regard serum NPY (data are presented as mean + SD and range)

\begin{tabular}{|l|c|c|c|cc|}
\hline & Group I N=30 & Group II N=30 & T value & P-value & ignificance \\
\hline NpY (ng/L) & $438.333 \pm 26.850$ & $91.500 \pm 8.613$ & 8.557 & $<0.001^{*}$ & HS \\
\hline
\end{tabular}

$\mathrm{HS}=$ Highly-significant, Serum NPY is higher patients group (group I) than in control group (group II) with high statistically significant difference.

Table (2): Comparison between laboratory results of both Groups Ia and Ib NPY (data are presented as mean \pm SD and range)

\begin{tabular}{|c|c|c|c|c|c|}
\hline & Group Ia $(\mathbf{N}=30)$ & Group Ib $(\mathrm{N}=30)$ & T value & P-value & Significance \\
\hline $\mathrm{Cr}(\mathrm{mg} / \mathrm{dL})$ & $2.047 \pm 0.770$ & $2.383 \pm 0.919$ & -3.910 & $0.001^{*}$ & $\mathrm{~S}$ \\
\hline Blood urea (mg/dL) & $60.433 \pm 4.143$ & $70.767 \pm 6.904$ & -4.177 & $<0.001^{*}$ & HS \\
\hline 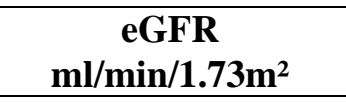 & $36.900 \pm 7.851$ & $31.373 \pm 7.852$ & 2.345 & $0.026^{*}$ & $S$ \\
\hline NPY (ng/dL) & $438.333 \pm 26.850$ & $630.667 \pm 64.926$ & -3.435 & $0.002 *$ & $\mathrm{~S}$ \\
\hline $\mathrm{Na}(\mathrm{mEq} / \mathrm{dL})$ & $134.967 \pm 5.762$ & $138.100 \pm 4.901$ & -2.340 & $0.026^{*}$ & $\mathrm{~S}$ \\
\hline $\mathrm{K}(\mathbf{m E q} / \mathbf{d L})$ & $4.369 \pm 0.491$ & $4.246 \pm 0.453$ & 1.614 & 0.117 & $\mathrm{NS}$ \\
\hline $\mathrm{Ca}(\mathrm{mg} / \mathrm{dL})$ & $8.310 \pm 0.634$ & $8.253 \pm 0.461$ & 0.647 & 0.523 & NS \\
\hline$P(\mathrm{mg} / \mathrm{dL})$ & $4.263 \pm 0.783$ & $4.290 \pm 0.540$ & -0.238 & 0.814 & NS \\
\hline $\begin{array}{c}\text { Serum albumin } \\
(\mathrm{g} / \mathrm{dL})\end{array}$ & $3.577 \pm 0.523$ & $3.605 \pm 0.403$ & -0.338 & 0.738 & NS \\
\hline Urinary PCR & $1.090 \pm 0.32$ & $1.013 \pm 0.16$ & 0.804 & 0.428 & NS \\
\hline WBC & $6.830 \pm 1.367$ & $6.517 \pm 1.085$ & 1.406 & 0.170 & NS \\
\hline Hb (g/dL) & $10.447 \pm 1.169$ & $10.753 \pm 0.710$ & -2.204 & $0.036^{*}$ & SS \\
\hline Platelets & $246.767 \pm 9.693$ & $249.867 \pm 6.235$ & -0.275 & 0.785 & NS \\
\hline
\end{tabular}

$\mathrm{NS}=$ Non-significant, $\mathrm{S}=$ Statistical significant, $\mathrm{HS}=$ highly significant $(\mathrm{p}<0.001)$. 
In table (2) there was significant increase in serum creatinine after follow up, highly significant increase in blood urea with time, significant decrease in eGFR after six months follow up and for serum NPY which increased significantly with time. Hemoglobin level increased with statistically significant difference, also serum $\mathrm{Na}$ increased with statistically significant difference.

Table (3): Comparison between group Ia and Ib CKD stages

\begin{tabular}{|c|c|c|c|c|c|c|c|}
\hline \multirow{2}{*}{ Stage } & \multicolumn{2}{|c|}{ Group Ia $(\mathrm{N}=30)$} & \multicolumn{2}{|c|}{ Group Ib $(\mathrm{N}=30)$} & \multicolumn{2}{|c|}{ Chi-Square } & \multirow{2}{*}{ SS } \\
\hline & $\mathbf{N}$ & $\%$ & $\mathbf{N}$ & $\%$ & $\mathrm{X}^{2}$ & P-value & \\
\hline Stage 1 & 1 & 3.33 & 0 & 0.00 & \multirow{7}{*}{9.031} & \multirow{7}{*}{0.108} & \multirow{7}{*}{ NS } \\
\hline Stage 2 & 0 & 0.00 & 2 & 6.67 & & & \\
\hline Stage 3a & 9 & 30.00 & 2 & 6.67 & & & \\
\hline Stage 3b & 6 & 20.00 & 7 & 23.33 & & & \\
\hline Stage 4 & 14 & 46.67 & 18 & 60.00 & & & \\
\hline Stage 5 & 0 & 0.00 & 1 & 3.33 & & & \\
\hline Total & 30 & 100.00 & 30 & 100.00 & & & \\
\hline
\end{tabular}

* NS= Non-significant, There was no statistically significant change in CKD stages after six months.

Table (4): ROC curve.

\begin{tabular}{|c|c|c|c|c|c|}
\hline \multicolumn{7}{|c|}{ ROC curve between Patients and Controls } \\
\hline Cutoff & Sensitivity & Specificity & PPV & NPV & Accuracy \\
\hline$>110$ & 100.0 & 93.33 & 93.7 & 100.0 & $97.7 \%$ \\
\hline
\end{tabular}

ROC curve shows the relationship between serum NPY and progression of CKD as regard patient group and control group.

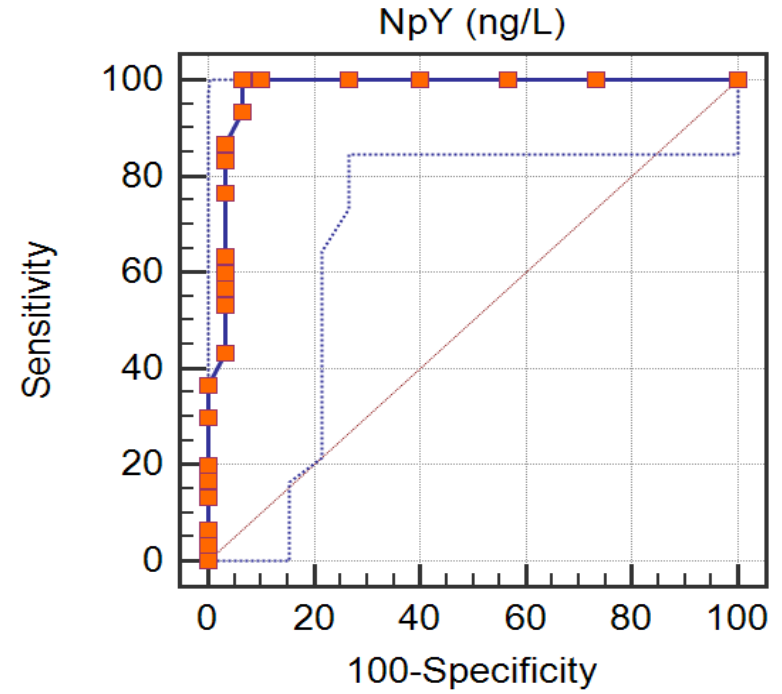

Fig. (1): ROC curve analysis.

ROC curve shows the relationship between serum NPY and progression of CKD as regard patient group and control group. The ROC curve was obtained to determine the sensitivity and specificity of serum NPY as a marker of progression in CKD patients and the best cut off point to detect CKD status was calculated with NPY greater than 110ng/L with sensitivity $100 \%$ and specificity $93.33 \%$. Area under the curve is 0.977

\section{DISCUSSION}

The first observation of the current study was that NPY levels were significantly higher in patients with CKD than in healthy controls. This was in accordance with the study of Zoccali et al. ${ }^{(3)}$ who aimed to test the hypothesis that neuropeptide is not only associated with CKD progression, but also play a role in the development of cardiovascular co-morbidities in CKD patients. They found that NPY levels were significantly elevated in patients with CKD. Moreover they had a conclusion that NPY level could be a predictor of cardiovascular events in CKD patients.

The study conducted by Klin and his colleagues also agreed with our results. They aimed to compare the NPY levels between CKD patients and healthy controls. They found, in agreement with the current study, that NPY levels were significantly higher in CKD patients together with some other plasma catecholamines and leucine-enkephalin ${ }^{(9)}$.

Another study that had results consistent with the present study is Hegbrant et $\boldsymbol{a l} .^{(\mathbf{1 0})}$ Although their study was conducted on hemodialysis patients unlike our study which was conducted on CKD patients not on dialysis, yet, they proved that NPY levels were higher in these patients. Odar et al. ${ }^{(11)}$ also linked high NPY levels to CKD patients and presence of volume induced hypertension in those patients.

The second finding of our study was that NPY levels were significantly higher in patients with CKD six months after the first measurement. This may suggest that NPY level is associated with progression of CKD. This was in partial accordance with the recent study of Zoccali et al. ${ }^{(1)}$ which was conducted in 2018. They investigated the relationship between circulating NPY and the progression of the GFR and proteinuria and the risk for a combined renal endpoint ( $>30 \%$ GFR loss, dialysis/transplantation) in two European CKD cohorts including follow-up of 753 and 576 patients for 36 and 57 months, respectively. They found that NPY was associated with the progression of the estimated GFR (eGFR) and proteinuria over time in both unadjusted and adjusted analyses by the mixed linear model. They also found that, in a combined analysis of the two cohorts accounting for the competitive risk of death (Fine and 
Gray model), NPY predicted $(\mathrm{P}=0.005)$ the renal endpoint (sub-distribution hazard ratio (SHR): 1.09; 95\% CI: $1.03-1.16 ; \mathrm{P}=0.005)$ and the SHR in the first cohort (1.14, 95\% CI: 1.04-1.25) did not differ $(\mathrm{P}=0.25)$ from that in the second cohort $(1.06,95 \% \mathrm{CI}$ : $0.98-1.15)$. Their conclusion was that NPY associates with proteinuria and faster CKD progression as well as with a higher risk of kidney failure. These findings suggest that the sympathetic system and/or properties intrinsic to the NPY molecule may play a role in CKD progression $^{(\mathbf{1})}$.

Another interesting finding of that study ${ }^{(1)}$ which was also in accordance to our results partially is that in both cohorts, NPY levels went along with the progression of proteinuria over time, the risk for faster GFR loss and renal events was virtually unaffected by statistical adjustment for proteinuria. This finding suggests that NPY may lead to kidney failure also by non-proteinuric pathways. In the present study, the proteinuria did not differ significantly after six months. Another study suggested that genetic variants of a biomarker of sympathetic activity like the chromogranin gene associate with a substantially non-proteinuric disease like nephrosclerosis ${ }^{(\mathbf{1 2})}$.

Although the aim of their work was different from our aim, Lu et al. ${ }^{(13)}$ agreed with the present study in that NPY levels increase with progression of CKD. They also linked between NPY levels and both nutritional sate and cerebrovascular co-morbidities in CKD patients.

This was in contrary to the study of Zoccali $\boldsymbol{e t}$ $\boldsymbol{a l}^{(\mathbf{1})}$ who found that NPY levels were significantly higher in elder CKD patients and reported a significant positive correlation between NPY and age. They also found that NPY levels increased along with progression of proteinuria in a significant positive correlation.

Bald et $\boldsymbol{a l}^{\left({ }^{(14)}\right.}$ also disagreed with our results as they showed that there was a significant negative correlation between the NPY level and GFR in both children and adults with CKD.

In our study we found that serum $\mathrm{Na}$ increased with statistically significant difference after six months which comes in agreement with the interesting study of Cole and his colleagues, as they found an association between higher serum sodium concentration and subsequent eGFR decline in people with established CKD when they collected data in a retrospective cohort study over a six year period and found that this association occurred while the serum sodium was in its normal range ${ }^{(\mathbf{1 5})}$.

The final finding in our study was increased hemoglobin level with statistically significant difference with deterioration of eGFR. This is in a disagreement with Yamamoto and his colleagues. Their study found that hemoglobin level decreases with the progression of CKD which increases the risk of cardiovascular diseases $^{(16)}$.

\section{CONCLUSION}

In this case-control study, increased serum NPY was associated with deterioration of eGFR, serum creatinine and blood urea as a consequent of CKD progression but at the same time serum NPY cannot be a marker for proteinuria progression as urinary $\mathrm{PCR}$ was not significantly changed. The study time was too short to get more significant changes in CKD stages.

Authors' contributions: Saeed Abdel Wahab Saeed and Haitham Ezzat gave idea. Hend Ahmed Aboelsaad collected the patients' data and analyse them. Nahla Mohammed Teama put study design. Nahla Mohammed Teama and Hend Ahmed Aboelsaad wrote the paper with revision. They all approved the final version of the manuscript.

\section{REFERENCES}

1. Zoccali C, D'arrigo G, Leonardis D et al. (2018): Neuropeptide $\mathrm{Y}$ and chronic kidney disease progression: a cohort study. Nephrol Dial Transpl., 33(10):1805-12.

2. Zoccali C, Mallamaci F, Tripepi G et al. (2003): Neuropeptide $\mathrm{Y}$, left ventricular mass and function in patients with end stage renal disease. J Hypertens., 21(7):1355-62.

3. Zoccali C, Mallamaci F, Tripepi G et al. (2003): Prospective study of neuropeptide $\mathrm{Y}$ as an adverse cardiovascular risk factor in end-stage renal disease. J Am Soc Nephrol., 14(10):2611-7.

4. Panuccio V, Cutrupi S, Pizzini P et al. (2007): Neuropeptide $Y$ and markers of osteoblast activity in dialysis patients: a crosssectional study. Am J Kidney Dis., 50(6):1001-8.

5. Morris MJ, Cox HS, Lambert GW et al. (1997): Regionspecific neuropeptide Y overflows at rest and during sympathetic activation in humans. Hypertension, 29(1):137-43.

6. Huber TB, Gloy J, Henger A et al. (1998): Catecholamines modulate podocyte function. J Am Soc Nephrol., 9(3):335-45.

7. Rafiq K, Noma T, Fujisawa Y et al. (2012): Renal sympathetic denervation suppresses de novo podocyte injury and albuminuria in rats with aortic regurgitation. Circulation, 125(11):1402-13.

8. Grassi G, Seravalle G, Ghiadoni L et al. (2011): Sympathetic nerve traffic and asymmetric dimethylarginine in chronic kidney disease. Clin J Am Soc Nephro., 6(11):2620-7.

9. Klin M, Waluga M, Rudka $R$ et al. (1998): Plasma catecholamines, neuropeptide $\mathrm{Y}$ and leucine-enkephalin in uremic patients before and after dialysis during rest and handgrip. Boll Chim Farm., 137(8):306-13.

10. Hegbrant J, Mårtensson $L$, Thysell $H$ et al. (1994): Changes in plasma levels of vasoactive substances during routine acetate and bicarbonate hemodialysis. Clin Nephrol., 41(2):106-12.

11. Odar-Cederlof I, Ericsson F, Theodorsson E et al. (1998): Is neuropeptide Y a contributor to volume-induced hypertension? Am J Kidney Dis., 31(5):803-8.

12. Salem RM, Cadman PE, Chen Y et al. (2008): Chromogranin A polymorphisms are associated with hypertensive renal disease. J Am Soc Nephrol., 19(3):600-14.

13. Lu L, Zou YC, Wang M et al. (2015): Neuropeptide Y levels are associated with nutritional status and cardiovascular events in adults with chronic kidney disease. Eur J Clin Nutr., 69(6):71721.

14. Bald M, Gerigk M, Rascher W (1997): Elevated plasma concentrations of neuropeptide $\mathrm{Y}$ in children and adults with chronic and terminal renal failure. Am J Kidney Dis., 30(1):23-7.

15. Cole NI, Suckling RJ, Desilva V et al. (2019): Serum sodium concentration and the progression of established chronic kidney disease. J Nephrol., 32(2): 259-264.

16. Yamamoto T, Miyazaki M, Nakayama M et al. (2016): Impact of hemoglobin levels on renal and non-renal clinical outcomes differs by chronic kidney disease stages: the Gonryo study. Clin Exp Nephrol., 20(4):595-602. 